\title{
Speech/Non-Speech Detection for Electro-Larynx Speech Using EMG
}

\author{
Anna Katharina Fuchs, Clemens Amon and Martin Hagmüller \\ Signal Processing and Speech Communication Laboratory, Graz University of Technology, Inffeldgasse 16/EG, Graz, \\ Austria \\ anna.fuchs@tugraz.at, clemens.amon@zoho.com,hagmueller@tugraz.at
}

Keywords: $\quad$ Speech/Non-Speech Detection (SND), Electro-Larynx (EL), Electromyography (EMG)

\begin{abstract}
Electro-larynx speech (EL) is a possibility to re-obtain speech when the larynx is surgically removed or damaged. As currently available devices normally are hand-held, a new generation of EL devices would benefit from a hands-free version. In this work we use electromyographic (EMG) signals to investigate speech/nonspeech detection for EL speech. The muscle activity, which is represented by the EMG signal, correlates with the intention to produce speech sounds and therefore, the short-term energy can serve as a feature to make a speech/non-speech decision. We developed a data acquisition hardware to record EMG signals using surface electrodes. We then recorded a small database with parallel recordings of EMG and EL speech and used different approaches to classify the EMG signal into speech/non-speech sections. We compared the following envelope calculation methods: root mean square, Hilbert envelope, and low-pass filtered envelope, and different classification methods: single threshold, double threshold and a Gaussian mixture model based classification. This study suggests that the results are speaker dependent, i.e. they strongly depend on the signal-to-noise ratio of the EMG signal. We show that using low-pass filtered envelope together with double threshold detection outperforms the rest.
\end{abstract}

\section{INTRODUCTION AND RELATED WORK}

In Germany there are around 21000 people without larynx and about 3000 additional laryngectomy surgeries are performed every year (Schiefer and Hagen, 2000). According to (Shute, 2003) the most popular device is the Servox Inton, which has no possibility to control on and off automatically. This kind of device normally is tube like and fits into one hand. While speaking, people have to hold it against the neck and press a button to turn the device on and off. Therefore, many electro-larynx (EL) user would benefit from a new EL device which can be controlled in a hands-free manner. We assume that the muscles which control the larynx are partially unimpaired. Hence, using the EMG signals is a possibility to detect speech and non-speech parts. We can use this information to control on and off messages of the EL device.

Our work is strongly encouraged by previous efforts to deal with speech/non-speech detection (SND) for EL speech (Goldstein et al., 2004), (Pineda-Rico et al., 2008): In (Goldstein et al., 2004) the EMG signal was band-pass filtered $(10-500 \mathrm{~Hz})$, amplified, rectified, and low-pass filtered (1-9 Hz corner fre- quency) for the creation of envelopes that tracked EMG activity. This approach was implemented in an EMG-EL prototype and reaction time experiments were conducted and compared to conventional pushbutton EL and normal voice. The developed analog version of the EMG-EL device had a controllable activation threshold. This single threshold was set to a value of $10 \%$ of the amplitude range. The termination threshold was based on an internal (fixed) activation-threshold-dependent hysteresis band. The optimized threshold using recorded sentences and a minimal cost function was not significantly different than the threshold they choose in their informal tests. In follow up work a new wireless version of the EMGEL was presented (Heaton et al., 2011). Within this work the EL could be either manually controlled or automatically using the EMG signal (same processing as described above). Later, the same group used a computer based system with two controllable thresholds (double threshold) (Kubert et al., 2009). In this work it is confirmed that EMG signals can serve as an intuitive and effective control source for EL voice activation and termination as well as $f_{0}$ modulation.

In (Ooe, 2012) the authors confirmed these findings. They used absolute values of the EMG signals. After smoothing with a moving average filter, 
a single threshold converts the envelope into the activation/termination signal. The time delay between EMG signal and speech start/stop was measured and it was confirmed that EMG signals can be used to control on/off signals for the EL device.

Also (Pineda-Rico et al., 2008) picks up the EMG based SND approach. They implemented a switching capacitor CMOS based device. For activation and termination the same method as in (Heaton et al., 2011) was taken: amplified, rectified and low-pass filtered $\left(c_{f}=3 \mathrm{~Hz}\right)$ envelope and single threshold implemented as voltage comparator. Their focus was on the implementation and on the advantages of switching capacitor circuits which are: excellent time constants, relative precision, simple design elements, minimum power waste and reduced size on chip.

In this work we developed an EMG signal acquisition hardware to capture EMG signals and recorded a database consisting of EL speech and EMG signals. We employed different strategies to smooth the EMG envelope and developed a threshold based method (single and double) and a statistical method (GMMs) to detect voice activity and evaluate its performance.

\section{METHODS}

\subsection{Data Acquisition Hardware}

We developed a data acquisition hardware in order to reduce costs and size. The requirements for the biosignal acquisition system were to be small, batteryoperated and real-time capable. It consists of three main parts: the sensor straps, the bio-signal shield and an ARDUINO $^{(\mathcal{C}}$ DUE micro-controller board. The board serves as a host for the connected strap and the shield (see Figure 1).

The strap is designed to be worn around the neck to ensure correct electrode position at the surface of the sternohyoid muscle. This muscle is a long, thin muscle which is located along the length of the front of the human neck. The functions of this muscle include, depression of the hyoid bone, head and neck movement, and speech. This position is often used in Automatic Speech Recognition and the relation between muscle movement and fundamental frequency was confirmed (Ooe et al., 2010). The strap holds three silver/silver-chloride electrodes, two of them are used to detect the EMG signal, the third one serves as a reference electrode to improve the common-mode rejection ratio. The strap is connected to the instrumentation amplifier which is followed by an operational amplifier. The gain of this amplifier can be modified manually. After a low-pass filter where high frequency noise is suppressed, the positive and negative half-wave are split and fed to two discrete ana$\log$ inputs of the micro-controller. Using this method, a higher bit resolution (i.e. 13 bit) of the digitized signal amplitude can be achieved. Then the signal is converted from analog to digital. In the following experiments the micro-controller board is connected to the computer via USB which serves to power the shield via the micro-controller board $(5 \mathrm{~V})$. The sampling rate $f_{s}$ of the ARDUINO ${ }^{\complement}$ DUE ADC is set to 8 $\mathrm{kHz}$. This is enough as most of the frequency content of EMG signals is between 0 and $500 \mathrm{~Hz}$.

The authors are aware that in a real-world application, the algorithms need to be implemented on a DSP and the power supply and the hardware needs to be summarized such as it can be worn on the body, e.g. in a pocket.

\subsection{Recorded Database}

To evaluate different approaches for SND a database was recorded and simulations were done off-line using the recordings.

We used around 100 phonetically balanced speech utterances of a female and a male speaker. The skin surface EMG sensor are positioned around the neck and are attached to our processing hardware. EMG and speech signals are recorded using both, the biosignal shield connected to an audio interface (RME Fireface 800), and a head-mounted microphone AKG HC 577L with omni-directional pickup pattern. The audio interface ensures a high quality digital signal. The sampling rate of the audio interface was set to $44100 \mathrm{~Hz}$. Compared to the used sound card (24 bit), the micro-controller system is able to convert the input signal with a resolution of 13 bit. This is enough to perform all processing steps which are proposed in this work without drawbacks in respect to signal detection. We analyzed the recordings manually and annotated speech and non-speech sections in order to obtain a ground truth.

All in all we recorded $18 \min 45 \mathrm{~s}$ of data. The mean signal-to-noise ratio (SNR) for the male EMG signals was $16.7 \mathrm{~dB}$ and for the female $12.6 \mathrm{~dB}$. For SNR calculations we used first order IIR smoothing. This difference in SNR will also influence the thresholds for SND. The main energy of the EMG signal is between $0 \mathrm{~Hz}$ and $500 \mathrm{~Hz}$, in fact, over $90 \%$ of the energy can be found in this range. The ratio of speech to non-speech in the database was $63 \%$ to $37 \%$. 


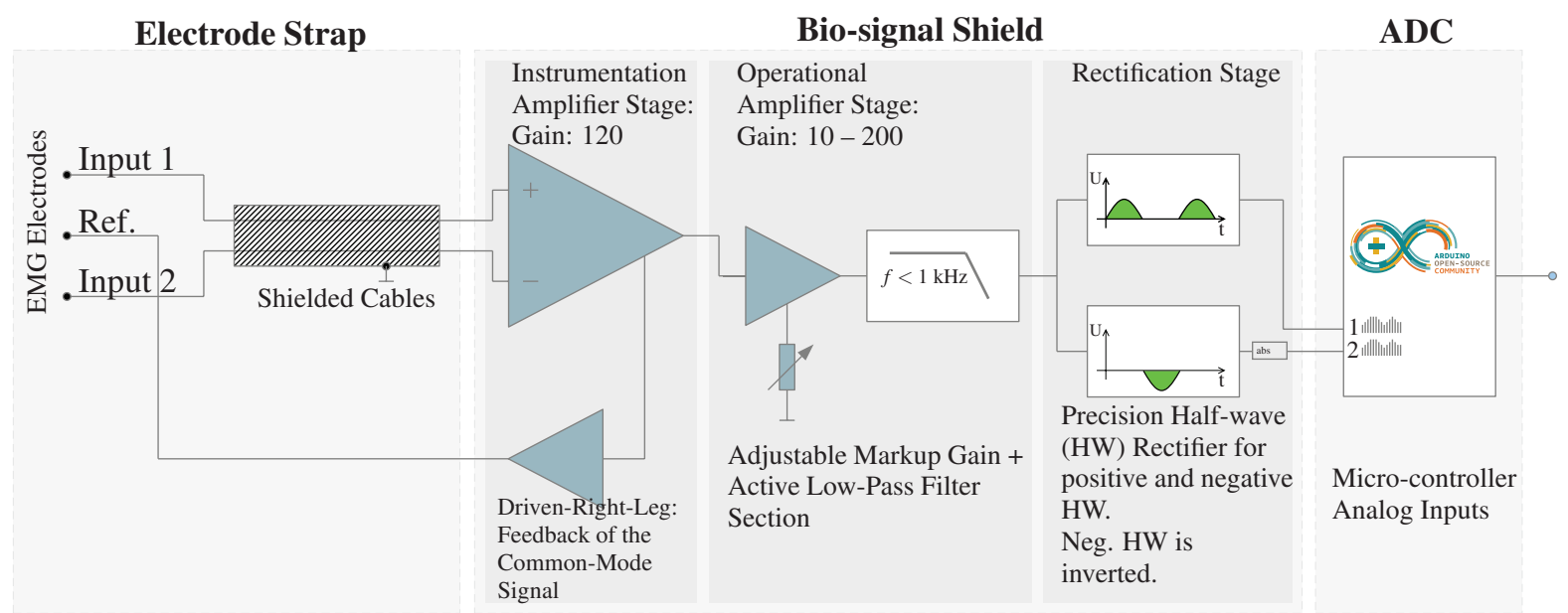

Figure 1: Schematic overview: Block diagram of the developed hardware system consisting of the electrode strap, the biosignal shield which is compatible to the ARDUINO ${ }^{\complement}$ DUE micro-controller which serves as a host.

\subsection{Pre-processing, Envelope Calculation, Speech/Non-Speech Detection (SND)}

In order to clean and prepare the recorded EMG signal some pre-processing needed to be applied. We used adaptive noise cancellation to remove crosstalk of the EL excitation signal and the EMG signal. Afterward a notch filter reduces interferences from electric hum caused by magnetic fields close to the sensors and amplification unit. The database was split into three parts in order to perform 3-fold cross validation. The sentences were chosen randomly for each validation set.

We choose three different envelope calculation methods: 1) root mean square (RMS) , 2.) Hilbert transform and 3.) low-pass (LP) filtered signal which was also suggested by (Goldstein et al., 2004).

1) Root mean square: In EMG analysis, the RMS of the signal is a common envelope calculation method to get the power of the signal. The RMS value for a windowed signal $x$ with window length $N$ is defined as $\operatorname{RMS}\{x\}=\sqrt{\frac{1}{N} \sum_{n=1}^{N} x[n]^{2}}$. 2) Hilbert transform: Another method to get the power of the EMG signal is to use the Hilbert transformed signal and rectify it. If we compare the Hilbert envelope to a simply rectified EMG signal, it can be seen that this method works as an amplitude follower and provides, even un-smoothed, an envelope which is not touching the zero line on the y-axis. Both, RMS and Hilbert envelope use a moving average filter for smoothing. This filter can cause oscillation and leakage into the smoothed output due to the sharp edges in the rectangular weighting function. A latency related to the length of the window is expected in live applications.

3) Low-pass filter (LP): The signal is rectified and a low-pass filter with a corner frequency of $5 \mathrm{~Hz}$ is ap- plied. A LP smooths the envelope. Implemented as a 3-pole IIR filter it produces a potential delay of up to $150 \mathrm{~ms}$. If a finite impulse response filter is built the time domain response must be time truncated, therefore it can be replaced by a moving average where the delay time is specified by the block size.

Using the different envelope calculation methods, we want to find SND messages using a) single threshold (ST), b) double threshold (DT) and c) classification using Gaussian mixture models (GMM).

Classification using a) single threshold and b) double threshold is straight forward. The calculated envelope is compared with a threshold. As soon as the envelope exceeds this threshold speech is detected and vice verse, when the envelope falls below the threshold we determine the message for non-speech (see Figure 2 - upper plot). In case of the double threshold, speech is detected when the envelope surpasses the first threshold and non-speech is detected if the thresholds drops below the second threshold (see Figure 2 - lower plot). A GMM is fully described with the parameters $\lambda=\left(b_{m}, \mu_{m}, \sigma_{m}\right) ; m=1,2, \ldots K$. $\mu$ is the mean value, $\sigma$ the variance and $b$ the weights for each of the $K$ components $m$. For the GMM SND two GMMs are trained, one for speech and one for non-speech. The number of components was determined using the Bayes information criterion and lies between 8 and 32. In the test we compute the probability density function for an unknown input for both GMMs and the maximum classifies into the SND results. We trained the parameters $(b, \mu$ and $\sigma)$ on two validation sets and tested on the third. All combinations assured that we use all utterances in the test. 

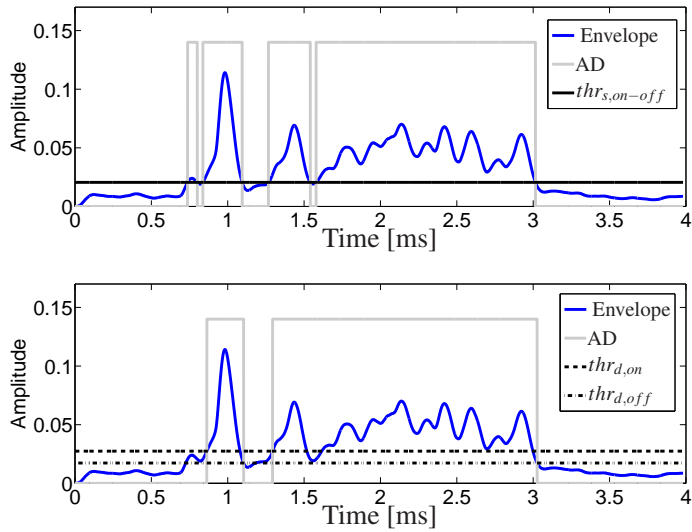

Figure 2: Activity detection (AD) using single threshold (upper plot) and double threshold (lower plot); Note that $t h r_{s, o n-o f f}$ is equal to $t h r_{d, o n}$.

\section{EXPERIMENTAL EVALUATION}

\subsection{Threshold Determination}

Single threshold as well as double threshold for SND were determined using receiver operating characteristics (ROC). In ROC curves sensitivity (true positive rate) is plotted against 1-specificity (false positive rate). The rates are calculated analyzing the database sentences with thresholds going from $1 \%$ to $100 \%$ in steps of $1 \%$. For single threshold the activation threshold $t h r_{s, o n-o f f}$ (= termination threshold) is based on the maximal envelope amplitude, for double threshold the termination threshold $t h r_{d, o f f}$ is relative to the activation threshold $t h r_{d, o n}$.

Under the assumptions of signal detection theory, we interpolate the ROC points of the different values of the threshold. These thresholds for best detection (highest sensitivity in combination with highest specificity) are calculated by finding the threshold, where the sum of the sensitivity and the absolute value of the specificity minus 1 has a maximum. The thresholds were chosen gender dependent.

\subsection{Error Calculation}

The SND results are compared to the speech ground truth. Errors are calculated regarding the correctly detected activation time and taking into account the interruptions of the detection. The absolute error is classified in the following parameters (Freeman et al., 1989): front end error (FEE), back end error $(B E E)$, middle speech error (MSE) and noise detected as speech $(N D S)$ (see Figure 3). In order to get error values which are comparable the 4 error types are normalized to the total length of the analyzed sentence.

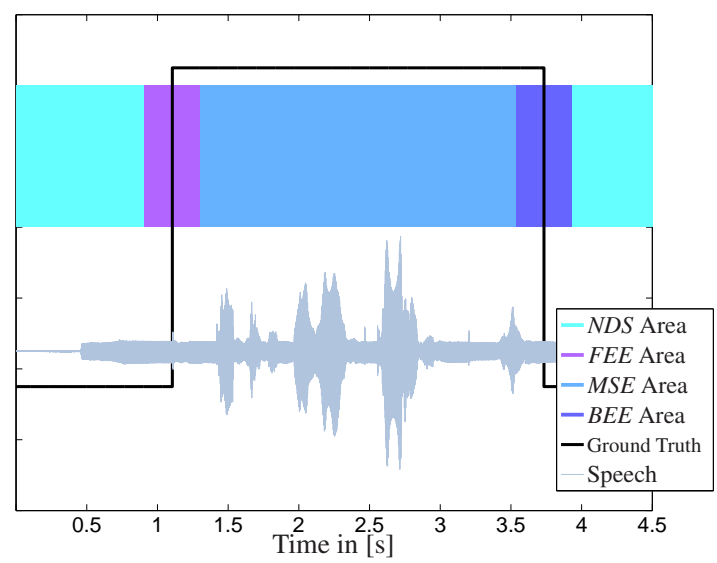

Figure 3: Error regions for noise detected as speech (NDS), front end error $(F E E)$, mid speech error (MSE) and back end error $(B E E)$ of an EL sentence.

We assume that MSE and FEE are the most important errors. If there is no triggering when the person wants to say something, information is lost. This error influences the speech quality much more than unwanted triggering when the person does not want to say anything, which, of course, is annoying and must also be avoided. The averaged absolute error for one method was calculated as the averaged sum of each separate error for a number of $N$ sentences $i$ :

$$
|\overline{e r}|=\frac{1}{N} \sum_{i=1}^{N} F E E_{i}+B E E_{i}+M S E_{i}+N D S_{i} .
$$

Moreover, the relation between the correct number of interruptions inside a sentence and the unwanted interruptions due to a wrong behavior of the detection process is presented as an indicator for jitter of the SND. The block detection ratio $(B D R)$ is defined as the ratio between the number of active blocks in the detection vector and the desired number of blocks in the ground truth vector. A perfect detection in terms of this interruption indicator would result in a block detection ratio of 1 .

\subsection{Time Constant Detection Smoothing}

For post-processing of short-time SND, it is common to apply detection smoothing or long time detection algorithms to the detection output. This is done to avoid short interruptions of the detection. In live scenarios this is problematic as for offset events the length of the following interruption cannot be predicted. The proposed time constant detec- 
tion smoothing algorithm treats two problems: 1. detection results might suffer from short interruptions and 2. EMG during speech shows a pre-activation of about $40 \mathrm{~ms}$ compared to the speech signal (Atkinson, 1978). The algorithm is using a time constant to smooth the detection output in a real-time application and therefore avoids small interruptions of detection and, at the same step, the algorithm is compensating EMG pre-activation. The time constant detection smoothing is delaying every on- and offset by the time constant $40 \mathrm{~ms}$.

\subsection{Experimental Results}

\subsubsection{Threshold Determination}

Single thresholds $t h r_{s, o n-o f f}$ for the female are lower than for the male speaker. The thresholds for LP and Hilbert envelope are similar, but for RMS it is lower: F: LP - 25\%, HIL - 24\%, RMS - 20\%; M: LP - 18\%, HIL - 17\%, RMS - 12\%. For double threshold the activation threshold $t h r_{d, o n}$ is higher than $t h r_{s, o n-o f f}$, the termination threshold $t h r_{d, o f f}$ is approximately the same for all envelope calculation strategies and both genders (see Table 1).

Table 1: Speech/Non-Speech thresholds for female (F) and male $(\mathrm{M})$ and for different envelope calculation methods: root mean square (RMS), Hilbert transform (HIL) and lowpass filtered (LP); $t h r_{d, o n}$ in \% based on maximal envelope, $t h r_{d, o f f}$ in $\%$ based on the activation threshold $t h r_{d, o n}$ (see subsection 3.1).

\begin{tabular}{|c|c|c|c|c|}
\hline & $\mathrm{F}$ & & $\mathrm{M}$ & \\
\hline \hline & $t h r_{d, o n}$ & $t h r_{d, o f f}$ & $t h r_{d, o n}$ & $t h r_{d, o f f}$ \\
\hline \hline RMS & $26 \%$ & $66 \%$ & $18 \%$ & $53 \%$ \\
HIL & $32 \%$ & $67 \%$ & $21 \%$ & $60 \%$ \\
LP & $33 \%$ & $63 \%$ & $25 \%$ & $60 \%$ \\
\hline
\end{tabular}

Sensitivity was between $88 \%$ and $96 \%$ and the specificity between $77 \%$ and $89 \%$. This means that in each method around $90 \%$ was detected correctly and the false positive rate (1-specificity: speech detected, no speech in ground truth) was up to $20 \%$ which leads to algorithms with very good performance.

\subsubsection{Error Analysis}

The individual error results and the block detection ratio values are shown in Figure 4 for the female speaker and in Figure 5 for the male speaker. The averaged absolute error $|\overline{e r}|$ together with the standard deviation $(s)$ and the $95 \%$ confidence interval $\left(C_{95 \%}\right)$ for the mean are presented in Table 2 . The presented results are processed with the proposed time constant detection smoothing (see subsection 3.3). It was possible to improve $|\overline{e r}|$ around $3 \%$ where especially FEE and $M S E$ was reduced. The time constant detection smoothing also improved the block detection ratio.

- $|\overline{e r}|$ for the male speaker $(6.4 \%-12.0 \%)$ are better than for the female speaker $(9.5 \%-12.2 \%)$.

- Double threshold outperforms single threshold for male and female speaker.

- LP envelope outperforms the RMS and Hilbert envelope in all tested scenarios (Single threshold, double threshold and GMM based approach) except for female HIL-GMM which slightly outperforms $(0.1 \%)$ LP-GMM.

- In GMM classification the MSE and NDS reaches low values but the FEE errors are increased.

- GMM is comparable with LP-DT for female speaker. For male speaker LP-GMM is the best method but there are huge differences between the GMM methods.

- Block detection ratio $B D R$, a measure for interruptions, results are improved with the time constant detection smoothing and present good results for both speaker (ca. 3 for female; ca. 2 for male).

With our tuned parameters, LP envelopes with double threshold perform best for female (second: HILGMM) and LP envelopes with GMM for male speaker (second: LP-DT).
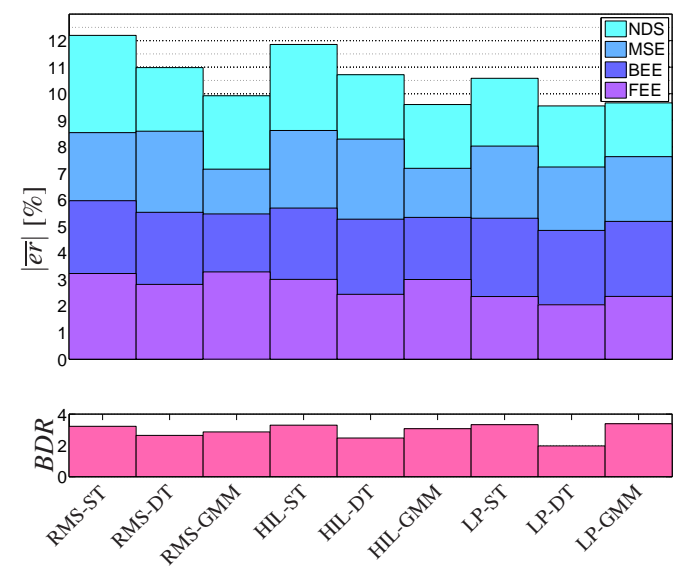

Figure 4: FEMALE: Averaged absolute error $|\overline{e r}|$ for all envelope calculation strategies: (root mean square (RMS), Hilbert envelope (HIL) and low-pass filter (LP) together with classification methods: single threshold (ST), double threshold (DT) and GMM (upper plot); block detection ratio $(B D R)$ (lower plot). 
Table 2: Averaged absolute error $|\overline{e r}|$ for female (F) and male (M); Best and second best methods in light Grey (female) and dark Grey (male); * indicates significant difference to the best method (F: LP-DT; M: LP-GMM).

\begin{tabular}{|c|c|c|c|c|}
\hline \multirow{2}{*}{ Method } & \multicolumn{2}{|c|}{$|\overline{e r}|(\mathrm{s})[\%]$} & \multicolumn{2}{c|}{$C I_{95}[\%]$} \\
\cline { 2 - 5 } & $\mathrm{F}$ & $\mathrm{M}$ & $\mathrm{F}$ & $\mathrm{M}$ \\
\hline \hline RMS-ST & $12.2(6.7)$ & $12.0(6.3)$ & $10.9-13.5 *$ & $10.8-13.2 *$ \\
RMS-DT & $11.0(6.8)$ & $11.5(6.8)$ & $9.7-12.3$ & $9.7-12.3 *$ \\
HIL-ST & $11.9(6.8)$ & $9.9(5.7)$ & $10.6-13.1 *$ & $8.8-10.9 *$ \\
HIL-DT & $10.7(6.6)$ & $8.7(6.7)$ & $9.5-12.0$ & $7.5-10.0 *$ \\
LP-ST & $10.6(5.3)$ & $7.8(4.9)$ & $9.6-11.6$ & $6.9-8.7 *$ \\
LP-DT & $9.5(5.6)$ & $6.6(4.7)$ & $8.5-10.6$ & $5.7-7.4$ \\
RMS-GMM & $9.9(5.7)$ & $9.1(5.3)$ & $8.9-11.0$ & $8.1-10.1 *$ \\
HIL-GMM & $9.6(5.5)$ & $8.0(5.0)$ & $8.6-10.6$ & $7.1-8.9 *$ \\
LP-GMM & $9.7(5.6)$ & $6.4(5.8)$ & $8.6-10.7$ & $5.3-7.5$ \\
\hline
\end{tabular}

\section{DISCUSSION AND CONCLUSIONS}

This works investigates a preliminary strategy in order to perform SND based on EMG signals which are insensitive to background noise. We compared different envelope calculation methods (RMS, HIL, LP) and classification strategies (ST, DT, GMM) to control the SND. In future the best approach should be implemented in a real-time system to control an EL device. In such a system the methods have to be optimized concerning the middle speech error and noise detected as speech error.

The thresholds were chosen gender dependent, because they change with the SNR. The SNR was lower for the female speaker than for the male speaker. This corresponds to the findings in (Goldstein et al., 2004) which say, that the amplitude of the EMG signal depends on the pitch; the lower the pitch, the larger the
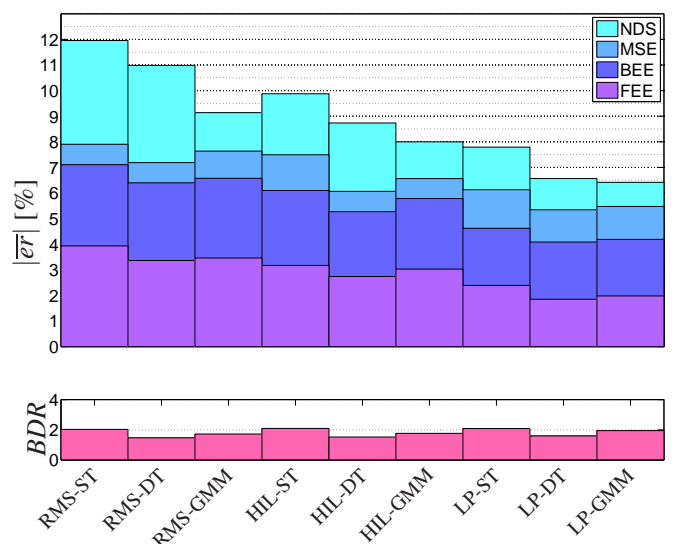

Figure 5: MALE: Averaged absolute error $|\overline{e r}|$ for all envelope calculation strategies: (root mean square (RMS), Hilbert envelope (HIL) and low-pass filter (LP) together with classification methods: single threshold (ST), double threshold (DT) and GMM (upper plot); block detection ratio $(B D R)$ (lower plot).
EMG amplitudes.

The different envelope calculation methods differ in how smooth they become. The smoothing time is one of the limiting factors in real-time because we can only look a certain time into the future. The proposed Hilbert envelope method (HIL) has the advantage that it incorporates an amplitude follower and therefore includes a smoothing. In our experiments Hilbert envelope performs better than RMS and about the same than LP for low SNRs. For high SNRs LP clearly outperforms RMS and Hilbert envelope. Furthermore, Hilbert envelope incorporates a moving average filter which might be an advantage over the low-pass filter (LP) because of the attack time of the 3-pole IIR filter.

GMMs suffer from the fact that their might be differences in the conditions for the training utterances and for the test. We can avoid this problem when we apply an adaptation strategy in the real-time scenario (Reynolds et al., 2000).

In our experiments a low-pass filtered version of the EMG signals together with a double threshold detection or a GMM classifier outperform other methods.

In order to do real-time smoothing, a time constant detection smoothing was implemented. It takes advantage of the preceding effect and smooths fast variations in the detected signal. This especially improves $F E E$ errors, because the activation is shifted in time.

For future work we have to consider several things: 1. Using EMG signals, non-speech related muscle movements like gulping will trigger the EL device. We assume that within a learning phase such unwanted events can be avoided. 2. We must keep in mind that the feedback during real-world test could (and will) influence the neck muscle activity and the resulting EMG behavior. In future work we will investigate the learning effect. 3 . We did not investigate the difference of EMG signals for healthy subjects and larygectomees. 4. The analysis was performed subject dependent. We can not talk about gender de- 
pendent differences because we only evaluated signals for 2 subjects, i.e. both gender.

We developed a data acquisition hardware for EMG signals which can serve as a prototype in a future real-time implementation. We found out that Hilbert envelope and LP envelope together with double threshold and GMM yield the best results in terms of error calculation. Due to the strong dependency on the SNR the thresholds need to be adjustable during speaking. The time constant detection smoothing turned out to be a good method for smoothing SND results and can also be implemented in real-time.

\section{ACKNOWLEDGMENTS}

The authors would like to thank HEIMOMED Heinze $\mathrm{GmbH} \& \mathrm{Co} . \mathrm{KG}$ for their support.

\section{REFERENCES}

Atkinson, J. E. (1978). Correlation analysis of the physiological factors controlling fundamental voice frequency. The journal of the Acoustical Society of America, 63(1):211-222.

Freeman, D., Cosier, G., Southcott, C., and Boyd, I. (1989). The voice activity detector for the pan-european digital cellular mobile telephone service. In International Conference on Acoustics, Speech, and Signal Processing, pages 369-372.

Goldstein, E. A., Heaton, J. T., Kobler, J. B., Stanley, G. B., and Hillman, R. E. (2004). Design and implementation of a hands-free electrolarynx device controlled by neck strap muscle electromyographic activity. Biomedical Engineering, IEEE Transactions on, 51(2):325-332.

Heaton, J., Robertson, M., and Griffin, C. (2011). Development of a wireless electromyographically controlled electrolarynx voice prosthesis. In Annual International Conference of the IEEE Engineering in Medicine and Biology Society, EMBC, pages 53525355.

Kubert, H., Stepp, C., Zeitels, S.M. anad Gooey, J., Walsh, M., Prakash, S., Hillman, R., and Heaton, J. (2009). Electromyographic control of a hands-free electrolarynx using neck strap muscles. Journal of communication disorders, 42(3):211-225.

Ooe, K. (2012). Development of controllable artificial larynx by neck myoelectric signal. Procedia Engineering, 47(0):869 - 872. 26th European Conference on Solid-State Transducers.

Ooe, K., Villagran, C., and Fukuda, T. (2010). Development of the compact control system using of neck emg signal for welfare applications. In International Symposium on Micro-NanoMechatronics and Human Science (MHS), pages 127-132.
Pineda-Rico, Z., Dieck-Assad, G., Martinez-Chapa, S., and Avila-Ortega, A. (2008). A switching capacitor cmos based device for hands-free electrolarynx activation using electromyographic signals. In Electronics, Robotics and Automotive Mechanics Conference, pages 8-13.

Reynolds, D. A., Quatieri, T. F., and Dunn, R. B. (2000). Speaker verification using adapted gaussian mixture models. Digital signal processing, 10(1):19-41.

Schiefer, J. and Hagen, R. (2000). Rehabilitation laryngektomierter Karzinompatienten. Der Onkologe, 6(1):36-43.

Shute, B. (2003). Perceptions of Artificial Larynx Reliability According to Laryngectomees and Speechlanguage Pathologists. Gonzaga University, Spokane, Washington.

\section{ACRONYMS}

$\begin{array}{ll}\text { BDR } & \text { Block Detection Ratio } \\ \text { BEE } & \text { Back End Error } \\ \text { DT } & \text { Double Threshold } \\ \text { EL } & \text { Electro-Larynx } \\ \text { EMG } & \text { Electromyography } \\ \text { FEE } & \text { Front End Error } \\ \text { GMM } & \text { Gaussian Mixture Model } \\ \text { HIL } & \text { Hilbert Transform } \\ \text { IIR } & \text { Infinite Impulse Response } \\ \text { LP } & \text { Low-Pass } \\ \text { MSE } & \text { Mid Speech Error } \\ \text { NDS } & \text { Noise Detected as Speech } \\ \text { RMS } & \text { Root Mean Square } \\ \text { ROC } & \text { Receiver Operating Characteristics } \\ \text { SND } & \text { Speech/Non-Speech Detection } \\ \text { SNR } & \text { Signal-to-Noise Ratio } \\ \text { ST } & \text { Single Threshold }\end{array}$

